\title{
Valgus stability is enhanced by flexor digitorum superficialis muscle contraction of the index and middle fingers
}

\author{
Shota Hoshika ${ }^{1,2}$, Akimoto Nimura $^{3^{*}}$, Norimasa Takahashi ${ }^{1}$, Hiroyuki Sugaya $^{1}$ and Keiichi Akita ${ }^{2}$
}

\begin{abstract}
Background: Flexor digitorum superficialis (FDS) muscle provides dynamic stabilization and medial elbow support for ulnar collateral ligament (UCL). The FDS contraction significantly affects the medial joint distance (MJD) through grip contraction. However, it remains unclear whether FDS activity alone contributes to medial elbow stability, or together with the activation of the flexor digitorum profundus during grip contraction, and which finger's FDS is the main contributor to elbow stability. We investigated the resistive effects of isolated FDS contraction in individual fingers against valgus stress in the elbow joint using stress ultrasonography (US).

Methods: We investigated 17 healthy males (mean age, $27 \pm 5$ years). Valgus stress US was performed using the Telos device, with the elbow at $30^{\circ}$ flexion. MJD was measured for each arm during 3 separate conditions: at rest (unloaded), under valgus load (50 N) (loaded), and under valgus load with FDS contracted in individual fingers (loaded-contracted).

Results: MJD was significantly longer when loaded $(5.4 \pm 0.4 \mathrm{~mm})$ than unloaded $(4.1 \pm 0.2 \mathrm{~mm}, P=0.007)$ or loadedcontracted ( $4.6 \pm 0.3 \mathrm{~mm}, P=0.003)$ for each finger. When loaded-contracted, MJD differed statistically between the index and ring fingers $(P=0.03)$ and between the middle and ring fingers $(P=0.04)$. However, the difference between the index and middle fingers was not statistically significant $(P=0.08)$.

Conclusions: Individual FDS contraction, particularly of the index and middle fingers, contributes most to stabilization against valgus stress. Thus, injury care programs should incorporate FDS exercises of these fingers.
\end{abstract}

Keywords: Ulnar collateral ligament, Ultrasound, Flexor digitorum superficialis, Overhead sports

\section{Background}

The prevalence of ulnar collateral ligament (UCL) injury and resultant operations in young baseball pitchers and professionals is increasing disturbingly [1, 2]. In order to avoid reconstruction surgery and its associated year-long recovery, improvement of preventive care programs is necessary [2]. The anterior bundle of the UCL is the primary restraint to valgus load at the medial elbow [3]. During the throwing motion, the tensile load on the

\footnotetext{
*Correspondence: nimura.orj@tmd.ac.jp

${ }^{3}$ Department of Functional Joint Anatomy, Graduate School of Medical and Dental Sciences, Tokyo Medical and Dental University, Tokyo, Japan

Full list of author information is available at the end of the article
}

UCL has been estimated to exceed its failure strength, leading to injury [4]. The flexor-pronator muscles (FPMs) are secondary dynamic stabilizers and have been considered to exert a protective effect against UCL injury [5-13]. However, the preventive arm care programs did not assess the effect of the finger flexors $[14,15]$.

Anatomical studies indicate that the FPMs lie in a good position to protect the UCL [6, 8]. Biomechanical studies report that the flexor digitorum superficialis (FDS) muscle plays the greatest role among the FPMs as an active stabilizer against valgus stress $[10,11,13,16]$. Ultrasonographic (US) studies report that the FDS has significant 
effects on medial joint distance (MJD) through grip contraction [14, 17]. However, there have been concerns relating to the assessment of FDS activity. First, it remains unclear whether FDS activity alone contributes to medial elbow stability, or together with the activation of the flexor digitorum profundus (FDP) during grip contraction [18]. Second, it remains unclear which finger's FDS contributes most to elbow stability.

This study aimed to evaluate MJD at rest, under valgus load, and under valgus load with isolated FDS contraction of each finger using stress US. We hypothesized that FDS activity alone contributes to medial elbow stability, and that FDS contraction in one specific finger provides dynamic stability against valgus stress.

\section{Methods}

\section{Design}

This study was a repeated measures analysis to assess changes in MJD during separate elbow loading conditions. The dependent variable was MJD, and the independent variables were the loading conditions and FDS contraction of each finger. The participants' dominant and nondominant elbows were subjected to rest (unloaded), valgus load (loaded), and valgus load with individual FDS contraction (loaded-contracted). These conditions were tested by separate examinations of the index, middle, and ring fingers.

\section{Participants}

Seventeen physically active individuals aged 22-38 years (mean age, $27 \pm 5$ years) were recruited within our institute from December 2017 to November 2018. Only male participants were enrolled, to mimic the state of an adult male baseball player. Participants with any of the following were excluded: (1) current pain or injury in the upper extremities, (2) previous UCL tear or elbow dislocation, (3) previous surgery in the upper extremities, and (4) previous participation in overhead sports. Institutional review board approval was obtained and written informed consent was obtained from all participants prior to enrollment.

\section{Imaging technique}

All US scans were performed by an experienced orthopedic surgeon (SH) using a SNIBLE (KONICA MINOLTA, Chiyoda, Japan) scanner with an 11-MHz linear transducer. Each participant was placed in a sitting position on a chair with the shoulder at $60^{\circ}$ abduction, the elbow at $30^{\circ}$ flexion, and the forearm in supination, similar to that in previous research (Fig. 1a) [19, 20]. All angles were measured using a goniometer, and the transducer was placed on the oblique coronal plane to visualize the MJD; this method is sensitive enough to identify an increase in the MJD when a valgus load is placed on the arm (Fig. 1b) [19-21].

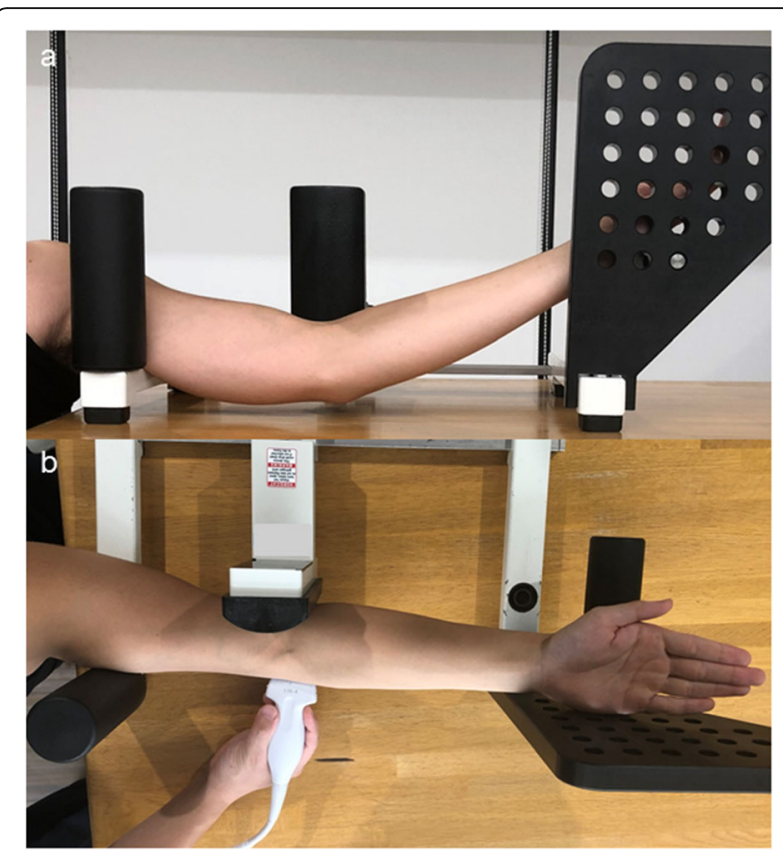

Fig. 1 The positioning of the elbow on the Telos stress device. a Subject sitting on a chair with the shoulder at $60^{\circ}$ abduction, the elbow at $30^{\circ}$ flexion, and the forearm in a supination position. $\mathbf{b}$ The ultrasonographic images are obtained from the medial side of the elbow. $50 \mathrm{~N}$ of valgus stress is applied to the lateral side of the elbow by the Telos device

Stress ultrasonographic examination across the 3 loading conditions

The stress US examination was used to assess the contribution of the FDS contraction of each finger to medial elbow joint stability. The MJD was measured as the distance between the distal-medial corner of the trochlea of the humerus and the proximal edge of the sublime tubercle of the ulna on the oblique coronal image (asterisks in Fig. 2). This was measured on the US screen with the use of electronic calipers with a precision of $0.1 \mathrm{~mm}$. Fixed valgus stress $(50 \mathrm{~N})$ was applied using a standardized instrumented device (Telos, Marburg, Germany) to the lateral side of the elbow joint to strain the medial aspect of the elbow (Fig. 1b). This valgus stress of $50 \mathrm{~N}$ was selected for two reasons: (1) some participants were unable to tolerate stress of over $50 \mathrm{~N}$ and were uncomfortable and (2) this amount of force has been suggested as appropriate for the Japanese population [22]. Using these established methods of collecting images and applying valgus stress, we were able to collect the measurements $(\mathrm{mm})$ of the unloaded, loaded, and loaded-contracted testing conditions [14].

Under the unloaded condition, the participant was placed in the testing position with no valgus stress applied to the elbow joint. The participant was asked to relax completely before an image was taken. Under the loaded condition, the participant was asked to relax 


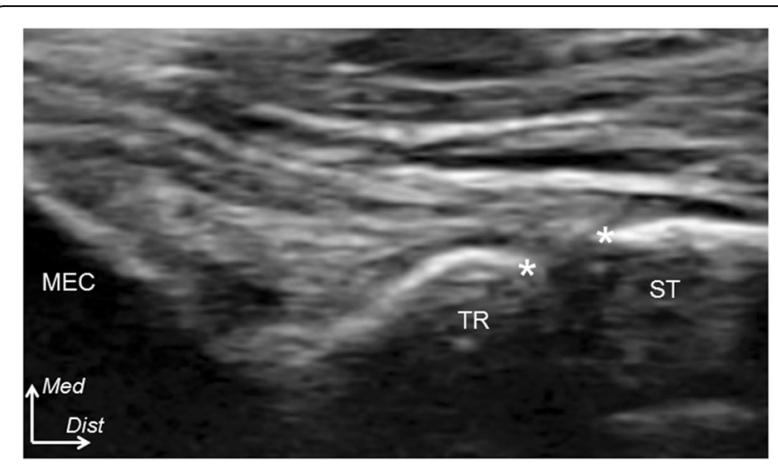

Fig. 2 An oblique coronal ultrasonographic examination of the humeroulnar joint. The medial joint distance is measured as the distance between the distal-medial corner of the trochlea (TR) and the proximal edge of the sublime tubercle (ST) (asterisks), in millimeters, from the images in three loading conditions: unloaded, loaded, and loaded-contracted. MEC, medial epicondyle; Med, medial; Dist, distal

while the valgus load was applied, and another image was taken. As a transition took place between the loading conditions, the valgus load was fully removed from the elbow to prevent excessive stress. A 1-min transition period was allowed between the testing conditions.

In the loaded-contracted condition, the participant was asked to flex the specified finger and maintain it at a fixed angle of $90^{\circ}$ at the proximal interphalangeal (PIP) joint (Fig. 3). To eliminate the effect of FDP contraction, we ensured that the finger was kept free of any tension at the distal interphalangeal (DIP) joint. With the finger in the flexed position, the FDS was activated, leading to its isometric contraction. The final image was taken while the load was applied during muscle contraction. No participants experienced elbow pain during the examination. Data for three trials of the separate loading conditions in each finger were collected and averaged for data analysis. We randomly tested the dominant and non-dominant arms and tested the index, middle, and ring fingers in that order. For interrater reliability, the MJD was evaluated twice at $>4$-week intervals. Interrater reliabilities were established and maintained, with interclass correlation coefficients in the acceptable range for all measures (0.90-0.98).

\section{Statistical analysis}

A one-way repeated measures analysis of variance (ANOVA) was performed to assess changes in the MJD for the separate loading conditions in each finger. When the ANOVA indicated statistical significance, a Bonferroni $t$ test was performed. In addition, a paired $t$ test was applied to compare the MJD between the dominant and non-dominant arms for each finger. A two-sided $P$ value of $<0.05$ was considered to indicate statistical significance. All analyses were performed with IBM SPSS Statistics 18 software for Windows (IBM Japan, Inc.). A power analysis for the detection of differences between separate loading conditions in each individual finger was conducted using an $\alpha$ value of 0.05 , an effect size of 0.4 (which was determined according to the results of a preliminary study), and a power of 0.95 . The power analysis suggested that 34 elbows were needed to assess the three separate loading conditions for each finger.

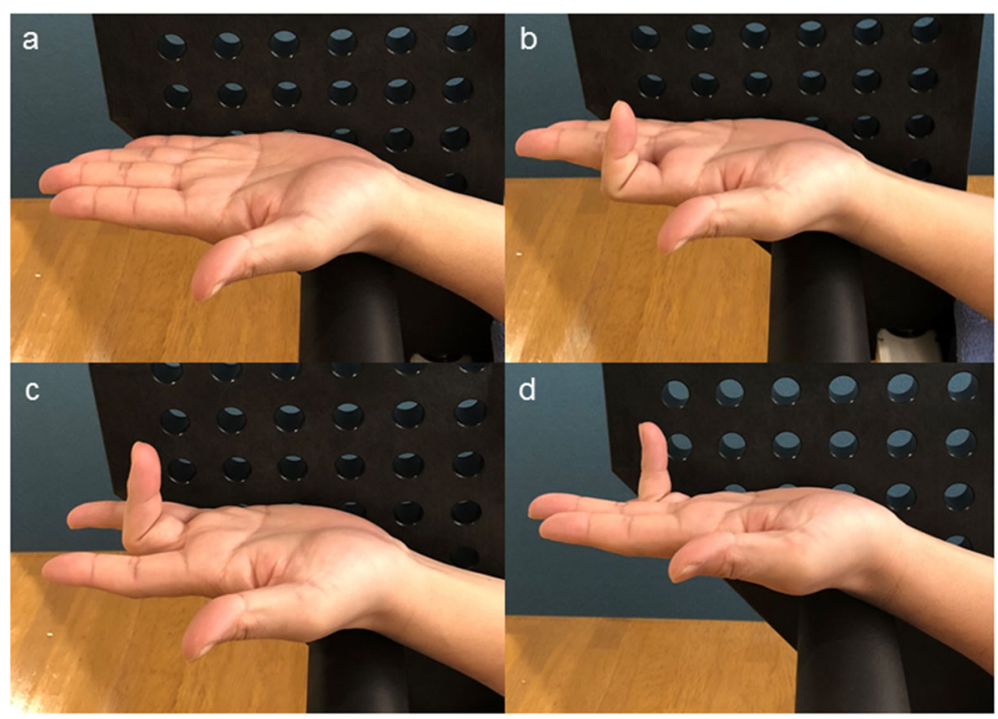

Fig. 3 Anterolateral view of each finger for individual flexor digitorum superficialis (FDS) activity. a All fingers are kept straight, thus not contracting the FDS. b The index finger is flexed at a fixed angle of $90^{\circ}$ at the proximal interphalangeal joint and maintained straight and free from any muscle contraction at the distal interphalangeal joint; this is done to only activate the FDS and to eliminate the effect of flexor digitorum profundus contraction of the specific finger. The participants maintain the activation by keeping the finger bent. c The FDS activation of the middle finger. $\mathbf{d}$ The FDS activation of the ring finger 


\section{Results}

Figure 4 and Table 1 show the values of MJD for each finger and both elbows. MJD was significantly longer when loaded $(5.4 \pm 0.4 \mathrm{~mm})$ than unloaded $(4.1 \pm 0.2$ $\mathrm{mm}, P=0.007)$ or loaded-contracted $(4.6 \pm 0.3 \mathrm{~mm}, P=$ 0.003 ) (Fig. 4). When loaded-contracted, MJD showed no statistically significant difference between the dominant and non-dominant arms for any finger (Table 1). There was a statistical difference in the MJD between the index and ring fingers $(P=0.03)$, and also between the middle and ring fingers $(P=0.04)$, when loadedcontracted (Fig. 4). However, the difference in MJD between the index and middle fingers was not statistically significant $(P=0.08)$ (Fig. 4$)$.

\section{Discussion}

The most important findings of this study were that FDS activity alone contributes to medial elbow stability. In addition, the FDS muscles of the index and middle fingers provide more dynamic stability against valgus stress than that of the ring finger.

Grip strength is an important indicator of hand function, including the functions of the FDS and FDP muscles. A hand dynamometer is used to evaluate the maximum power of the total grip strength of all fingers [23]. However, the FDP is considered a primary finger flexor, generating greater gripping strength than the FDS [24]. A recent biomechanical study demonstrated that the contribution of the FDS and FDP to the grip strength depends on the hand dynamometer angulation and contact area of each finger [18]. In addition, it is difficult to accurately evaluate grip strength in each finger by using the dynamometer. Finally, the grip strength of all fingers could not be associated with the FDS function in each finger. In our study, to obtain

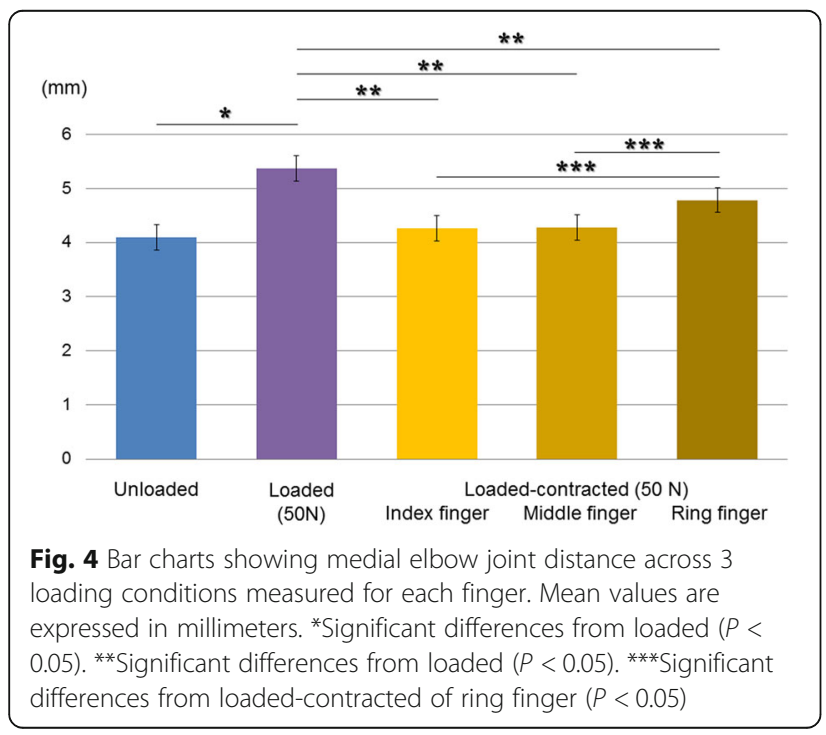

Table 1 The bilateral medial elbow joint distance in the loadedcontracted condition for each finger

\begin{tabular}{lllll}
\hline & & Dominant & Non-dominant & $P$ value \\
\hline Finger & Index & $4.3(4.0-4.6)$ & $4.3(4.0-4.6)$ & 0.59 \\
& Middle & $4.3(4.0-4.6)$ & $4.3(4.0-4.6)$ & 0.95 \\
& Ring & $4.8(4.6-5.0)$ & $4.8(4.4-5.2)$ & 0.70 \\
\hline
\end{tabular}

Values are expressed as millimeters (mean $(95 \% \mathrm{Cl})$ ). The locations of measurements are demonstrated in Fig. 2

isolated FDS activation, the subject was asked to flex the PIP joint while the DIP joint remained quiescent and not flexed in each finger [24]. Clearly, our study showed the contribution of isolated FDS function in each finger to the dynamics of the MJD.

Some anatomical studies reported that the FDS was the individual muscle best suited to provide medial elbow support because of its proximity and relatively large bulk among the FPMs $[6,8]$. Current in vitro research recognized that FDS activity had significant effects on MJD using stress US [14, 17]. However, these studies overlooked the importance of assessing FDS activity of individual fingers, as opposed to the activity in all of them collectively, through grip motion; thus, it remained unclear which finger's FDS contraction contributed most to elbow stability. This study demonstrates that FDS contraction of the index and middle fingers has a larger effect on MJD than that of the ring finger. This explains how FDS activation of the index and middle fingers has a primary role for stabilization of the medial elbow against valgus stress.

The stress US study showed that healthy volunteers displayed $0.7 \mathrm{~mm}$ of change in the MJD as measured with non-stress and stress [17]. Another study demonstrated that the change in the MJD under loaded and loadedcontracted conditions of the FDS was $0.2 \mathrm{~mm}$ [14]. Based on the previous studies, a minimum of $0.2 \mathrm{~mm}$ could be interpreted as a clinically significant difference in the loaded and loaded-contracted conditions compared to the unloaded condition. In the present study, the change in the MJD under loaded and loaded-contracted conditions of the isolated FDS compared to the unloaded condition was 1.3 and $0.8 \mathrm{~mm}$, respectively. Finally, the isolated FDS contraction could have clinically significant effects on the MJD to protect from valgus stress.

Previous studies have reported that the UCL of the dominant elbow in professional baseball pitchers was thicker than that of the non-dominant elbow and its thickness was associated with increased laxity against valgus stress [20, 25]. Recently, Hoshika et al. [26] identified that the anterior bundle of the UCL could be interpreted as part of the tendinous complex, which consisted of the tendinous septum (TS) and the FDS muscle. In other words, UCL thickness could be interpreted as the FDS muscle thickness. Given the anatomical fact, we could 
hypothesize that UCL thickness will change with medial elbow stabilization by the FDS contraction of each finger. Future research is needed to assess the changes in muscular thickness of the FDS with valgus stability by FDS contraction of each finger using the MJD through the stress US.

Based on these findings, an interpretation of the dynamic stabilizing effect of FDS function in these specific fingers can be described anatomically as follows: At the level of the mid forearm, the FDS muscles of the index and middle fingers are located on the radial side, and the ring and little fingers on the ulnar side. In addition, the FDS muscle of the index finger is located in the superficial layer and that of the middle finger in the deep layer [27]. Hoshika et al. [26] also reported that the tendinous complex might be a pathway of the muscular power of the FPMs to the humeroulnar joint. Taking these anatomical concepts into consideration, the FDS muscles of the index and middle fingers may connect with the TS; thus, these fingers could provide more dynamic stability against valgus stress than that of the ring finger via the TS.

Our study provides clinically relevant information that can be used in the development of a program to prevent UCL injury. Recently, there has been a focus on hand muscle training to prevent throwing injuries as well as to improve performance in generating ball velocity [14, 26, 28]. However, Thrower's Ten, which has a long history of use by overhead throwing athletes, incorporates multiple strengthening exercises for the shoulder, elbow, and wrist but fails to address the finger flexors [15]. Taking our current results into consideration, the preventive care program could be improved by incorporating finger exercises that focus particularly on the FDS muscles of the index and middle fingers. This study might aid the development of such programs.

The study has the following limitations: First, FDS activity of the little finger was not examined because it has no impact on throwing a ball. Second, we enrolled participants from the general population, and the medial elbow of baseball players may have adapted to chronic medial elbow stress and react differently. Future research in these athletes will allow the assessment of how these characteristics change in a sport-specific population. Third, we did not check any electromyography of the FDS and FDP muscles for the precise assessment of muscle activity in each finger, when cued to perform isolated FDS contraction without the effect of the FDP muscle. Given these limitations, this is the first study to assess MJD during FDS contraction in each finger.

\section{Conclusions}

This study reveals that individual FDS contractions, especially of the index and middle fingers, directly impact elbow dynamics more than that of the ring finger. Finger exercises that focus specifically on these muscles are important for preventing UCL injuries.

\section{Abbreviations \\ ANOVA: Analysis of variance; DIP: Distal interphalangeal; FDP: Flexor digitorum profundus; FDS: Flexor digitorum superficialis; FPMs: Flexor- pronator muscles; MJD: Medial joint distance; mm: millimeter; PIP: Proximal interphalangeal; TS: Tendinous septum; UCL: UInar collateral ligament; US: Ultrasonographic}

\section{Acknowledgements \\ Not applicable.}

\section{Authors' contributions}

TN and SH helped with the data collection, interpretation, and writing of the paper. HS prepared the manuscript. NA and AK designed the experiment.

The authors read and approved the final manuscript

\section{Funding}

This study was partly supported by a grant from JA Kyosai Research Institute (Agricultural Cooperative Insurance Research Institute).

\section{Availability of data and materials}

The datasets used and/or analyzed during the current study are available from the corresponding author on reasonable request.

\section{Ethics approval and consent to participate}

Our study has been approved by the Ethics Committee of the Funabashi Orthopaedic Hospital. The committee's reference number was 2018034.

\section{Consent for publication}

Consent for publication was obtained from all participants.

\section{Competing interests}

The authors declare that they have no competing interests

\section{Author details}

${ }^{1}$ Shoulder \& Elbow Service, Funabashi Orthopaedic Sports Medicine \& Joint Center, Funabashi, Chiba, Japan. '2Department of Clinical Anatomy, Graduate School of Medical and Dental Science, Tokyo Medical and Dental University, Tokyo, Japan. ${ }^{3}$ Department of Functional Joint Anatomy, Graduate School of Medical and Dental Sciences, Tokyo Medical and Dental University, Tokyo, Japan.

Received: 17 January 2020 Accepted: 20 March 2020

Published online: 30 March 2020

\section{References}

1. DeFroda SF, Goodman AD, Gil JA, Owens BD. Epidemiology of elbow ulnar collateral ligament injuries among baseball players: National Collegiate Athletic Association injury surveillance program, 2009-2010 through 20132014. Am J Sports Med. 2018;46:2142-7.

2. Rebolledo BJ, Dugas JR, Bedi A, Ciccotti MG, Altchek DW, Dines JS. Avoiding Tommy John surgery: what are the alternatives? Am J Sports Med. 2017;45: 3143-8.

3. Morrey BF, An KN. Functional anatomy of the ligaments of the elbow. Clin Orthop Relat Res. 1985:201:84-90.

4. Fleisig GS, Andrews JR, Dillman CJ, Escamilla RF. Kinetics of baseball pitching with implications about injury mechanisms. Am J Sports Med. 1995:23:233-9.

5. An KN, Hui FC, Morrey BF, Linscheid RL, Chao EY. Muscles across the elbow joint. A biomechanical analysis. J Biomech. 1981;14:659-69.

6. Davidson PA, Pink M, Perry J, Jobe FW. Functional anatomy of the flexor pronator muscle group in relation to the medial collateral ligament of the elbow. Am J Sports Med. 1995;23:245-50.

7. Digiovine NM, Jobe FW, Pink M, Perry J. An electromyographic analysis of the upper extremity in pitching. J Shoulder Elbow Surg. 1992;1:15-25.

8. Frangiamore SJ, Moatshe G, Kruckeberg BM, Civitarese DM, Muckenhirn KJ, Chahla J, Brady AW, et al. Qualitative and quantitative analyses of the 
dynamic and static stabilizers of the medial elbow: an anatomic study. Am J Sports Med. 2018;46:687-94.

9. Glousman RE, Barron J, Jobe FW, Perry J, Pink M. An electromyographic analysis of the elbow in normal and injured pitchers with medial collateral ligament insufficiency. Am J Sports Med. 1992;20:311-7.

10. Hamilton CD, Glousman RE, Jobe FW, Brault J, Pink M, Perry J. Dynamic stability of the elbow: electromyographic analysis of the flexor pronator group and the extensor group in pitchers with valgus instability. J Shoulder Elbow Surg. 1996;5:347-54.

11. Park MC, Ahmad CS. Dynamic contributions of the flexor-pronator mass to elbow valgus stability. J Bone Joint Surg Am. 2004;86:2268-74.

12. Sisto DJ, Jobe FW, Moynes DR, Antonelli DJ. An electromyographic analysis of the elbow in pitching. Am J Sports Med. 1987;5:260-3.

13. Udall JH, Fitzpatrick MJ, McGarry MH, Leba TB, Lee TQ. Effects of flexorpronator muscle loading on valgus stability of the elbow with an intact, stretched, and resected medial ulnar collateral ligament. J Shoulder Elbow Surg. 2009;18:773-8

14. Pexa BS, Ryan ED, Myers JB. Medial elbow joint space increases with valgus stress and decreases when cued to perform a maximal grip contraction. Am J Sports Med. 2018:46:1114-9.

15. Wilk KE, Meister K, Andrews JR. Current concepts in the rehabilitation of the overhead throwing athlete. Am J Sports Med. 2002;30:136-51.

16. Buffi JH, Werner K, Kepple T, Murray WM. Computing muscle, ligament, and osseous contributions to the elbow varus movement during baseball pitching. Ann Biomed Eng. 2015;43:404-15.

17. Otoshi K, Kikuchi S, Shishido H, Konno S. Ultrasonographic assessment of the flexor pronator muscles as a dynamic stabilizer of the elbow against valgus force. Fukushima J Med Sci. 2014;60:123-8.

18. Kaufmann RA, Kozin SH, Mirarchi A, Holland B, Porter S. Biomechanical analysis of flexor digitorum profundus and superficialis in grip-strength generation. Am J Orthop (Belle Mead NJ). 2007;36:128-32.

19. Ciccotti MC, Hammoud S, Dodson CC, Cohen SB, Nazarian LN, Ciccotti MG Stress ultrasound evaluation of medial elbow instability in a cadaveric model. Am J Sports Med. 2014;42:2463-9.

20. Ciccotti MG, Atanda A Jr, Nazarian LN, Dodson CC, Holmes L, Cohen SB. Stress sonography of the ulnar collateral ligament of the elbow in professional baseball pitchers: a 10-year study. Am J Sports Med. 2014;42: 544-51.

21. Bica D, Armen J, Kulas AS, Youngs K, Womack Z. Reliability and precision of stress sonography of the ulnar collateral ligament. J Ultrasound Med. 2015; 34:371-6.

22. Harada M, Takahara M, Maruyama M, Nemoto T, Koseki K, Kato Y. Assessment of medial elbow laxity by gravity stress radiography: comparison of valgus stress radiography with gravity and a Telos stress device. J Shoulder Elbow Surg. 2014;23:561-6.

23. Mathiowetz V, Weber K, Volland G, Kashman N. Reliability and validity of grip and pinch strength evaluations. J Hand Surg Am. 1984;9:222-6.

24. Long C 2nd, Conrad PW, Hall EA, Furler SL. Intrinsic-extrinsic muscle control of the hand in power grip and precision handling. An electromyographic study. J Bone Joint Surg Am. 1970;52:853-67.

25. Atanda A Jr, Buckley PS, Hammoud S, Cohen SB, Nazarian LN, Ciccotti MG. Early anatomic changes of the ulnar collateral ligament identified by stress ultrasound of the elbow in young professional baseball pitchers. Am J Sports Med. 2015;43:2943-9.

26. Hoshika S, Nimura A, Yamaguchi R, Nasu H, Yamaguchi K, Sugaya H, et al. Medial elbow anatomy: a paradigm shift for UCL injury prevention and management. Clin Anat. 2019;32:379-89.

27. Scott WW, William CP, Robert NH, Scott HK. Flexor tendon injury: Green's Operative Hand Surgery. 6th ed. Churchill Livingstone Elsevier; 2010.

28. Kinoshita H, Obata S, Nasu D, Kadota K, Matsuo T, Fleisig GS. Finger forces in fastball baseball pitching. Hum Mov Sci. 2017;54:172-81.

\section{Publisher's Note}

Springer Nature remains neutral with regard to jurisdictional claims in published maps and institutional affiliations.

Ready to submit your research? Choose BMC and benefit from:

- fast, convenient online submission

- thorough peer review by experienced researchers in your field

- rapid publication on acceptance

- support for research data, including large and complex data types

- gold Open Access which fosters wider collaboration and increased citations

- maximum visibility for your research: over $100 \mathrm{M}$ website views per year

At $\mathrm{BMC}$, research is always in progress.

Learn more biomedcentral.com/submissions 